\title{
Construcción y validación de un cuestionario para evaluar la percepción sobre la tutoría metodológica en los cursos de Especialización Médica
}

Construction and validation of a questionnaire to evaluate the perception of methodological tutoring in Medical Specialization courses

\author{
Raúl Hernández-Ordoñez ${ }^{1}$ (D) https://orcid.org/0000-0002-4060-1949 \\ Norma Amador-Licona ${ }^{1}$ (D) https://orcid.org/0000-0003-1470-6883 \\ ${ }^{1}$ Universidad De La Salle Bajío, Campus Campestre, ${ }^{凶}$ raul.hdzodz@outlook.com \\ (C) Universidad De La Salle Bajío
}

Recibido en: $02-10$ - 2020 / Aceptado en: $07-01-2020$

\section{Resumen}

Introducción: El tutor metodológico es concebido como una persona con experiencia investigativa, aportación de ideas constructivas, conocimientos e inclinado a colaborar con otras personas. Debe facilitar la realización de investigaciones relevantes, de alto nivel académico y compromiso social, sin perder de vista las relaciones interpersonales en los diversos escenarios y contextos.

Contar con un instrumento que permita evaluar la percepción de la tutoría metodológica puede beneficiar no solo a los tutores en la evaluación objetiva de su trabajo, sino a los coordinadores de educación para identificar áreas de oportunidad para el fortalecimiento de este proceso. El propósito de esta investigación es determinar la validez y confiabilidad del cuestionario para evaluar la percepción sobre la tutoría metodológica en los cursos de Especialización Médica.

Método: Se realizó un estudio cuantitativo con diseño transversal analítico en el Centro de Investigación Educativa y Formación Docente del Instituto Mexicano del Seguro Social, Delegación Guanajuato. Para la validez de contenido participaron cinco expertos. Para la prueba piloto se seleccionó una muestra no probabilística de 94 médicos residentes que cursaban el último año de la especialidad médica y que contaban con su proyecto de investigación concluido y registrado. La validez de contenido se determinó de forma cualitativa por ronda de expertos y cuantitativa, mediante el índice de Lowshe. La validez de constructo se realizó mediante el análisis factorial exploratorio (AFE) y la confiabilidad con el coeficiente de Alpha de Cronbach.

La investigación está apegada a los principios éticos establecidos en la Ley General de Salud y su Reglamento, así como en la Declaración de Helsinki en su última revisión. Se registró en el Comité Local de Investigación 1008 del IMSS. 
Resultados: Se determinó la validez de contenido por juicio de expertos, al considerar que el cuestionario es claro, suficiente, coherente y relevante. Asimismo, se obtuvo un Índice de Lowshe de 0.99. El coeficiente de alfa de Cronbach fue de 0.97 global ( 0.92 para competencias personales y socioemocionales y 0.96 en competencias para socializar y facilitar el proceso investigativo). El AFE permitió identificar una estructura de dos factores (con una medida de adecuación de tamaño de muestra KMO de 0.885, una prueba de esfericidad de Bartlet significativa [p=0.001] y una correlación entre las variables >0.6. El cuestionario está conformado por 21 ítems, con dos factores: Competencias personales y socioemocionales del tutor y competencias metodológicas para socializar y facilitar proceso investigativo, con 6 y 15 ítems respectivamente. Las competencias para la tutoría se agruparon en dos dimensiones que explicaron el $76.78 \%$ de las varianzas.

Conclusión: La psicometría del cuestionario es adecuada, por lo que cuenta con validez de contenido y de constructo, y con consistencia interna, por lo que permite evaluar la percepción de tutoría metodológica.

Palabras clave: tutoría metodológica; percepción; especialidad médica; tutores; residentes; confiabilidad; validez; medicina; salud; investigación; academia; formación; IMSS

\begin{abstract}
Introduction: The methodological tutor is conceived as a person with research experience, contribution of constructive ideas, knowledge and inclined to collaborate with other people. He or she should facilitate the carrying out of relevant research, of high academic level and social commitment, without losing sight of interpersonal relationships in various settings and contexts. Having an instrument that allows evaluating the perception of methodological tutoring can benefit not only tutors in the objective evaluation of their work, but also education coordinators to identify areas of opportunity to strengthen this process. The purpose of this research is to determine the validity and reliability of the questionnaire to assess the perception of methodological tutoring in Medical Specialization courses.

Method: A quantitative study with an analytical cross-sectional design was carried out at the Center for Educational Research and Teacher Training of the Instituto Mexicano del Seguro Social, Guanajuato Delegation. For content validity, five experts participated. For the pilot test, a nonprobabilistic sample of 94 resident physicians who were in the last year of the medical specialty and who had their research project concluded and registered were selected. The content validity
\end{abstract}


was determined qualitatively by expert round and quantitatively, using the Lowshe index. Construct validity was performed using exploratory factor analysis (EFA) and reliability, with the Cronbach's Alpha coefficient.

The research is attached to the ethical principles established in the General Health Law and its regulations, as well as in the Declaration of Helsinki in its latest revision. It was registered in the IMSS Local Research Committee 1008.

Results: The content validity was determined by expert judgment, considering that the questionnaire is clear, sufficient, coherent and relevant. Likewise, a Lowshe Index of 0.99 was obtained. Cronbach's alpha coefficient was 0.97 overall (0.92 for personal and socio-emotional competencies and 0.96 for competencies to socialize and facilitate the research process). The EFA allowed the identification of a two-factor structure (with a KMO sample size adequacy measure of 0.876, a significant Bartlet sphericity test $[\mathrm{p}=0.001]$ and a correlation between variables $>0.6$ ). The questionnaire was made up of 21 items, with two factors: Personal and socio-emotional skills of the tutor and skills to socialize and facilitate the research process, with 6 and 15 items respectively. The competences for tutoring were grouped into two dimensions that explained $76.78 \%$ of the variance.

Discussion or Conclusion: The psychometric of the questionnaire is adequate, so, it has content and construct validity, and internal consistency, which makes it possible to evaluate the perception of methodological tutoring.

Keywords: methodological tutoring, perception, medical specialty, tutors, residents, reliability, validity; medicine; health; research; academy; training; IMSS

\section{Introducción}

La principal labor del Instituto Mexicano del Seguro Social (IMSS) es la asistencia médica al derechohabiente, sin embargo, las actividades educativas (formación de médicos especialistas y de otro tipo de personal dedicado al cuidado de la salud), así como las actividades de investigación clínica y educativa, son esenciales para garantizar una atención de calidad. Se sabe que la aportación del IMSS a la formación de médicos especialistas lo ubica como la institución más importante en este terreno, tanto en México como en América Latina (Academia Mexicana de 
Cirugia, 2013). Es mediante los cursos de especialización médica, también conocidos como residencias médicas, que se lleva a cabo dicha formación.

Las residencias médicas comprenden la etapa de la educación médica de posgrado, en la cual los médicos desarrollan competencias profesionales especializadas, después de concluir la educación médica superior (licenciatura). Esta etapa formativa se desarrolla según las normas y regulaciones específicas federales e institucionales y se efectúa a partir de una estructura de aprendizaje, en la cual los médicos residentes están bajo la supervisión de especialistas con más experiencia, que asumen la responsabilidad de su formación e instrucción, a través de las siguientes actividades curriculares: practica-clínica en escenarios clínicos reales, supervisión de expertos, enseñanza teórica, evaluación sistemática y asesoría en la elaboración de proyectos de investigación (IMSS, 2012). Esta última, está incluida en su mapa curricular como asignatura, con la finalidad de: 1) desarrollar un sentido crítico que le permita identificar las fortalezas, debilidades y áreas de oportunidad en el proceso educativo; 2) desarrollar habilidades para la búsqueda y selección de información que favorezca la resolución de problemas y la mejor toma de decisiones, y 3) generar conocimientos a partir de proyectos de investigación, por lo que es obligatorio que realice al menos uno, durante su formación, al cual se le denomina "Tesis".

Varios cursos de especialización médica otorgados por el IMSS están inscritos en el Programa Nacional de Posgrados de Calidad del Consejo Nacional de Ciencia y Tecnología (CONACyT) y la Subsecretaría de Educación Superior de la Secretaría de Educación Pública. Los criterios de evaluación de este programa, exigen que, aunque los cursos de especialización médica sean profesionalizantes, para poder ser ubicados en el nivel "consolidado y de internacionalización", se debe generar conocimiento, mediante el desarrollo de proyectos de investigación.

Una forma de poder dar cumplimiento a este requisito es que cada uno de los residentes debe desarrollar al menos un proyecto de investigación durante sus años de residencia médica. Desafortunadamente, en la mayoría de los casos el trabajo de tesis representa el primer contacto del residente con una investigación bajo su propia responsabilidad (López Santiago et al., 2000; Castro Rodríguez et al., 2018), por lo que muchas de esas investigaciones no se verán culminadas con la difusión de sus resultados, ya sea en congresos, foros, publicaciones nacionales ni internacionales, ya que las investigaciones son más de interés personal y no hacia la resolución de problemas prioritarios de salud (Castro Rodríguez et al., 2018; Herrera-Márquez \& González- 
Cabello, 2015; Castro Rodríguez et al., 2018).

Es por lo anterior, que la figura del tutor, ya sea para la formación médica y/o en investigación resulta esencial. El asesor de tesis (tutor metodológico) ha sido concebido tradicionalmente como una persona con cierta experiencia investigativa, con aportación de ideas y sugerencias constructivas, disponibilidad de tiempo y conocimientos en determinada área, inclinado a colaborar con otras personas en la elaboración de investigaciones que le permitan cumplir con un requisito exigido por los estudios universitarios y de postgrados (Rosas et al., 2006; Guerra-Martín \& Borrallo-Riego, 2018).

Para poder brindar una adecuada tutoría, como primer requisito, el tutor debe ser un experto no solo en metodología de la investigación para la elaboración de proyectos, sino también en el proceso de tutoría, dirección y manejo de grupos, así como en el tema específico de investigación; además, se debe evitar la sobrecarga de trabajo, favorecer una adecuada relación y comunicación con los tutorados, en un ambiente y espacio favorable para el desarrollo de la asesoría (GuerraMartín \& Borrallo-Riego, 2018). El tutor debe ser un asesor, un mentor, un guía, y a la vez, un supervisor. Su trabajo es muy delicado porque debe saber qué y cómo orientar a sus tutorados sin suplantar su gestión, logrando su máxima independencia (Cruzata-Martínez et al., 2018).

Como se puede observar, la tutoría representa un mecanismo útil para personalizar y diversificar los procesos de aprendizaje. Se debe reconocer que es un factor determinante para potencializar el desarrollo del aprendizaje auto determinado (acceso a los conocimientos, el desarrollo de habilidades, hábitos y actitudes, y la internalización de valores), por lo que el tutor se constituye como mediador del aprendizaje, promueve en los estudiantes el estudio, el análisis crítico y la reflexión para que construyan sus aprendizajes de forma autónoma (Calixto Flores, 2016; Hamui-Sutton et al., 2018).

En el estudio de la tutoría, López Santiago et al. (2000) realizaron un estudio desde el enfoque cualitativo con el propósito de indagar sobre lo que los residentes esperan de sus tutores, lo que representa uno de los esfuerzos por conocer la función y percepción del tutor. Dentro de sus conclusiones resaltan: a) la confianza entre el tutor y el residente como un elemento esencial en la relación, b) un modelo de comunicación con criterios de igualdad entre dos individuos, que son a la vez adultos y profesionales, c) disponer de criterio y gobierno en la planificación de la formación, además de, d) considerar a los tutores como como modelos para la formación. 
De la Cruz Flores y García Campos (2006) realizaron una propuesta de un modelo integrador de la tutoría, en el que se identifican ocho funciones: a) formación en investigación, b) formación profesional, c) docencia, d) socialización, e) de entrenamiento, f) consejería académica, g) de patrocinador y h) de apoyo psicosocial. Cabe mencionar que este modelo es un modelo integrador, holístico. Su finalidad es promover una tutoría más consciente, reflexiva, favorecedora de la formación tanto académica-profesional como en investigación. Ellos integran la formación en investigación dentro de la tutoría académica global.

Haksever y Manisali (2000) indagaron la supervisión por parte del asesor en el posgrado, específicamente en el programa de doctorado en gestión e ingeniería de la construcción, e identificaron tres tipos de ayuda que el asesorado espera de su asesor, estas son definidas de la siguiente manera:

1) Ayuda personal: apoyo, motivación, socialización, ayuda para organizar el alojamiento y otras cosas que pueden ser necesarias, pero que no están relacionadas con la investigación.

2) Ayuda indirecta relacionada con la investigación: proporcionar contactos, tanto industriales como académicos, así como equipo.

3) Ayuda directa relacionada con la investigación: análisis crítico del trabajo y ayuda con problemas metodológicos.

Por lo tanto, el tutor de posgrado debe facilitar la realización de investigación relevante, de alto nivel académico y compromiso social, que contribuya a la solución de problemas nacionales. Durante la tutoría se establecen relaciones interpersonales, propiciando un sinnúmero de intercambios, académicos, personales, en diversos escenarios y contextos complejos. De todo esto depende, en gran medida, el éxito o fracaso académico del estudiante de postgrado (CruzataMartínez et al., 2018).

De acuerdo a lo antes descrito, es necesario puntualizar que, aunque las residencias médicas son cursos de posgrado a nivel de especialidad cuya finalidad es ser profesionalizantes más que de formación en investigación, ésta última no deja de ser de gran relevancia durante la formación, por sus implicaciones en la generación de conocimientos, el desarrollo de las mismas especialidades, así como en la prevención, tratamiento y mejora de la atención a la población, como se ha explicado previamente. Es por ello, que si bien, existen ya algunos instrumentos que permitan evaluar la tutoría académica en los cursos de posgrado como maestrías y doctorados, que serán revisados en 
detalle en la metodología, el objetivo del presente trabajo fue construir y validar un cuestionario que permitiera evaluar la percepción que tienen los médicos residentes sobre el proceso de la tutoría metodológica que reciben durante el desarrollo de los cursos de Especialización Médica para la elaboración de proyectos de investigación.

\section{Método}

\section{Diseño}

Se realizó un estudio con enfoque cuantitativo y diseño transversal analítico, para el cual se consideraron 4 etapas: a) determinación de la validez teórica, b) validez de contenido, c) coeficiente de confiabilidad y d) validez de constructo.

\section{Participantes}

Para la validez de contenido se seleccionaron cinco médicos, todos ellos con grado de maestría y con al menos un diplomado de formación en educación, además de que fueran o hayan sido tutores de tesis en los cursos de especialización médica. Para la determinación del coeficiente de confiabilidad de alpha de Cronbach y de la validez de constructo se realizó una prueba piloto en una muestra no probabilística por conveniencia de 96 médicos residentes, quienes habían recibido una tutoría metodológica y habían concluido su proyecto de investigación (para el tamaño de la muestra se consideraron los criterios propuestos por Osterlind (como fue citado por CarreteroDios \& Perez, 2005) quien menciona que para la muestra basta con que esté compuesta por entre 50 y 100 participantes. Así como lo referido por Ruiz (2013) quien menciona que se debe seleccionar una muestra de sujetos cuyo tamaño sea, como mínimo, equivalente al doble del número de ítems incluidos en el instrumento y que la muestra debe ser representativa.

\section{Construcción del instrumento}

Previo a tomar la decisión de construir y validar el cuestionario, se realizó una búsqueda bibliográfica en el Consorcio Nacional de Recursos de Información Científica y Tecnológica (CONRICyT) tanto en sus recursos abiertos como restringidos, así como en google académico, con la finalidad de identificar o encontrar un instrumento que permitiera evaluar la percepción de la tutoría metodológica por parte de tutorados; la búsqueda se realizó considerando diferentes tópicos: entre ellos: "tutoría", "funciones del tutor", "tutoría e investigación", "roles del tutor", 
"características de un tutor en el desarrollo de proyectos", "tutoría en investigación", "percepción de la tutoría", entre otros. De esta búsqueda, se identificaron algunos instrumentos, sin embargo, estos no atendían a las necesidades del presente estudio. Por ejemplo, De la Cruz y Abreu (2017) partiendo del modelo integrador de la tutoría, desarrollado por la primera autora, se embarcaron en la construcción y validación de un instrumento para evaluar la tutoría en los estudios de posgrado, orientados a la formación de investigadores (maestrías y doctorados). Dicho instrumento tiene un enfoque holístico, en el cual se evalúan todos los procesos formativos de estos cursos, está integrado en dos grandes dimensiones: Formativa-socializadora (Formación en investigación, formación profesional, docencia, entrenamiento, consejería académica, socialización, auspicio académico y apoyo social) y la dimensión interpersonal (Comportamiento ético, profesionalismo y clima de la interacción). Sin embargo, éste y otros instrumentos no atienden las necesidades del presente estudio, dado que la mayoría de los instrumentos no describen sus procesos de validación y confiabilidad; Además, en el caso del instrumento propuesto por De la Cruz y Abreu, este evalúa todo el proceso de la tutoría académica, en cursos de maestría y doctorado, y no únicamente el proceso que es de nuestro interés, por lo que se decidió construir un instrumento propio, tomando como base algunos de los instrumentos encontrados para desarrollar las categorías principales a evaluar de un asesor de proyectos de investigación.

Para la construcción de este instrumento se consideraron ítems de creación propia, así como algunos ítems de dos instrumentos diferentes: a) Construction management and engineering research supervision questionnaire - the student's viewpoint, de Mehmet Haksever y Manisali (2000) [ítems 1, 2, 3 y 8] y b) El Instrumento para Evaluar el Desempeño de la Tutoría, tomado de la ANUIES (2000) [ítems 4, 5, 7 y 13].

La tutoría metodológica comprende un proceso complejo, por lo que para facilitar su evaluación se consideraron inicialmente tres dimensiones que consideramos pertinentes de acuerdo con lo encontrado en la bibliografía, estas son: a) competencias personales y socio-emocionales; b) competencias para socializar y facilitar el proceso investigativo y; c) competencias metodológicas y de dirección; cada una de ellas se describe a continuación:

1) Competencias personales y socioemocionales: Se consideran todas aquellas acciones de apoyo, motivación y comunicación por parte del asesor, que favorecen la investigación, pero que no forman parte directamente ni con el proceso investigativo, ni con los aspectos metodológicos. 
2) Competencias para socializar y facilitar el proceso investigativo: se refiere a aquellas actividades encaminadas a facilitar y gestionar el uso de recursos, académicos y tecnológicos (equipos, material didáctico, software, ayuda inicial para localizar referencias), así como favorecer la interrelación con grupos académicos, de investigación u otros que favorezcan un mejor desarrollo de la investigación.

3) Competencias metodológicas y de dirección: ayuda directamente relacionada con aspectos metodológicos, análisis crítico del trabajo; así como la dirección y conducción precisa de la tesis.

\section{Análisis de la Validez del cuestionario}

La validez de contenido se determinó en forma cualitativa mediante juicio de expertos considerando un consenso en 4 de 5 expertos; y cuantitativamente a través del Índice de Lowshe. La validez de constructo se evaluó con el Análisis factorial exploratorio de Componentes principales; mientras que la fiabilidad se determinó mediante el coeficiente de Alfa de Cronbach tanto en forma global como para cada una de sus dimensiones.

\section{$\underline{\text { Resultados }}$}

Fue necesario llevar a cabo 3 rondas con los expertos para alcanzar el consenso buscado. Solo un ítem después de esta ronda no tuvo el consenso en 5 de 5 expertos, de tal manera que se obtuvo un índice de Lowshe de 0.99 , por lo que se consideró que el cuestionario cuenta con validez de contenido tanto cualitativa como cuantitativo.

Para la prueba piloto de los 110 cuestionarios entregados se recibieron 96, de los cuales se eliminaron dos, debido a que estaban contestados en forma incompleta, por lo que el número final de encuestados fue de 94. La edad media de los médicos residentes encuestados fue de $30.4 \pm 2.8$ años, con una proporción de $54.3 \%$ mujeres.

La evaluación de la consistencia interna se realizó mediante el análisis de fiabilidad Alpha de Cronbach, obteniendo un índice global de 0.97 (0.92 para las competencias personales y socioemocionales y 0.96 para las competencias para socializar y facilitar proceso investigativo); se evaluó la correlación ítem-total corregida no existiendo ningún puntaje menor de 0.3 que pudiera ser eliminado. Del instrumento propuesto inicialmente, se conservaron los cuatro ítems de "Construction management and engineering research supervision questionnaire - the student's 
Construcción y validación de un cuestionario para evaluar la percepción sobre la tutoría metodológica en los cursos de Especialización Médica

viewpoint"; mientras que de los ítems que se tomaron del "Instrumento para Evaluar el Desempeño de la Tutoría", se eliminó uno de ellos, por la similitud con el ítem (Resolvió satisfactoriamente cada una de las dudas que planteaste con relación a la tesis) considerando que este último es más general que el primero, además de que tiene un efecto negativo en los valores de las comunalidades de dos ítems del factor 2: a) Fue respetuoso y atento y b) Permitió que asumiera riesgos y tomara decisiones en el desarrollo de la tesis, incrementándose la comunalidad ante su eliminación de 0.500 para el a y 0.503 para el b respectivamente. En la Tabla 1 se muestran los resultados de la prueba.

Tabla 1. Resultado de Alpha de Cronbach (Estadísticos total-elemento).

Table 1. Result of Cronbach's Alpha (Total-element statistics).

\begin{tabular}{|c|c|c|c|c|}
\hline Ítem & $\begin{array}{l}\text { Media de la escala si } \\
\text { se elimina el } \\
\text { elemento }\end{array}$ & $\begin{array}{l}\text { Varianza de la escala } \\
\text { si se elimina el } \\
\text { elemento }\end{array}$ & $\begin{array}{l}\text { Correlación } \\
\text { elemento-total } \\
\text { corregida }\end{array}$ & $\begin{array}{l}\text { Alfa de Cronbach si } \\
\text { se elimina el } \\
\text { elemento }\end{array}$ \\
\hline Empleo frases de motivación & 91.266 & 236.477 & .585 & .975 \\
\hline Creo un clima de confianza & 90.883 & 242.040 & .631 & .974 \\
\hline $\begin{array}{l}\text { Favoreció una comunicación constante y } \\
\text { abierta }\end{array}$ & 90.936 & 237.544 & .736 & .973 \\
\hline Fue respetuoso y atento & 90.777 & 248.864 & .518 & .975 \\
\hline Mostró interés en problemas académicos & 90.947 & 238.416 & .725 & .973 \\
\hline $\begin{array}{l}\text { Permitió que asumieras riesgos y tomarás } \\
\text { decisiones en el desarrollo de la tesis }\end{array}$ & 90.915 & 241.692 & 640 & .974 \\
\hline Facilitó la relación con otras personas & 91.096 & 228.475 & .848 & .972 \\
\hline $\begin{array}{l}\text { Proporcionó información de buscadores, } \\
\text { software, material didáctico }\end{array}$ & 91.160 & 227.877 & .892 & .972 \\
\hline Orientó sobre bibliografía a consultar & 91.064 & 227.824 & .890 & .972 \\
\hline $\begin{array}{l}\text { Capacitó en el uso de software o } \\
\text { herramientas que facilitaran la elaboración } \\
\text { de la tesis }\end{array}$ & 91.245 & 226.208 & .857 & .972 \\
\hline $\begin{array}{l}\text { Proporcionó ayuda inicial para la } \\
\text { localización de referencias }\end{array}$ & 91.181 & 225.419 & .910 & .972 \\
\hline $\begin{array}{l}\text { Ayudó a registrar el protocolo en el } \\
\text { CLIES }\end{array}$ & 90.872 & 234.199 & .783 & .973 \\
\hline $\begin{array}{l}\text { Ayudó a realizar la difusión de resultados } \\
\text { en foros, congresos y/o publicaciones }\end{array}$ & 91.170 & 227.541 & .732 & .974 \\
\hline $\begin{array}{l}\text { Tiene capacidad para orientar en } \\
\text { metodología de la investigación }\end{array}$ & 90.979 & 231.182 & .871 & .972 \\
\hline $\begin{array}{l}\text { Muestra habilidades para el análisis } \\
\text { estadístico }\end{array}$ & 90.926 & 232.629 & .811 & .973 \\
\hline $\begin{array}{l}\text { Colaboró en la dirección y redacción del } \\
\text { escrito final }\end{array}$ & 91.021 & 229.698 & .765 & .973 \\
\hline $\begin{array}{l}\text { Fomentó el debate de ideas con el } \\
\text { asesorado }\end{array}$ & 91.000 & 229.591 & .906 & .972 \\
\hline Propició el análisis critico & 90.979 & 226.365 & .923 & .971 \\
\hline Domina el tema en el que asesoró & 90.989 & 228.118 & .826 & .972 \\
\hline $\begin{array}{l}\text { Resolvió satisfactoriamente cada una de } \\
\text { las dudas que planteaste con relación a la } \\
\text { tesis }\end{array}$ & 90.957 & 229.095 & .916 & .972 \\
\hline ¿Estás satisfecho con la asesoría recibida? & 90.915 & 228.573 & .895 & .972 \\
\hline
\end{tabular}

№ 26, Vol. 13 (2), 2021. ISSN 2007 - 0705, pp.: 1 - 17 
El análisis factorial exploratorio permitió identificar una estructura de dos factores (con una medida de adecuación de tamaño de muestra KMO de 0.876, una prueba de esfericidad de Bartlet significativa [2730.95, gl=201, p=0.001] y una correlación entre las variables >0.6).

Prueba de unidimensionalidad del constructo. De acuerdo con la regla de Kaiser, en el gráfico de sedimentación, al trazar una línea a nivel del autovalor 1, son dos los factores que explican la mayor parte de la variabilidad total (Gráfica 1).

Gráfica 1. Gráfico de sedimentación del cuestionario "Percepción del proceso de tutoría metodológica". Graph 1. Sedimentation graph of the questionnaire "perception of methodological tutoring process".

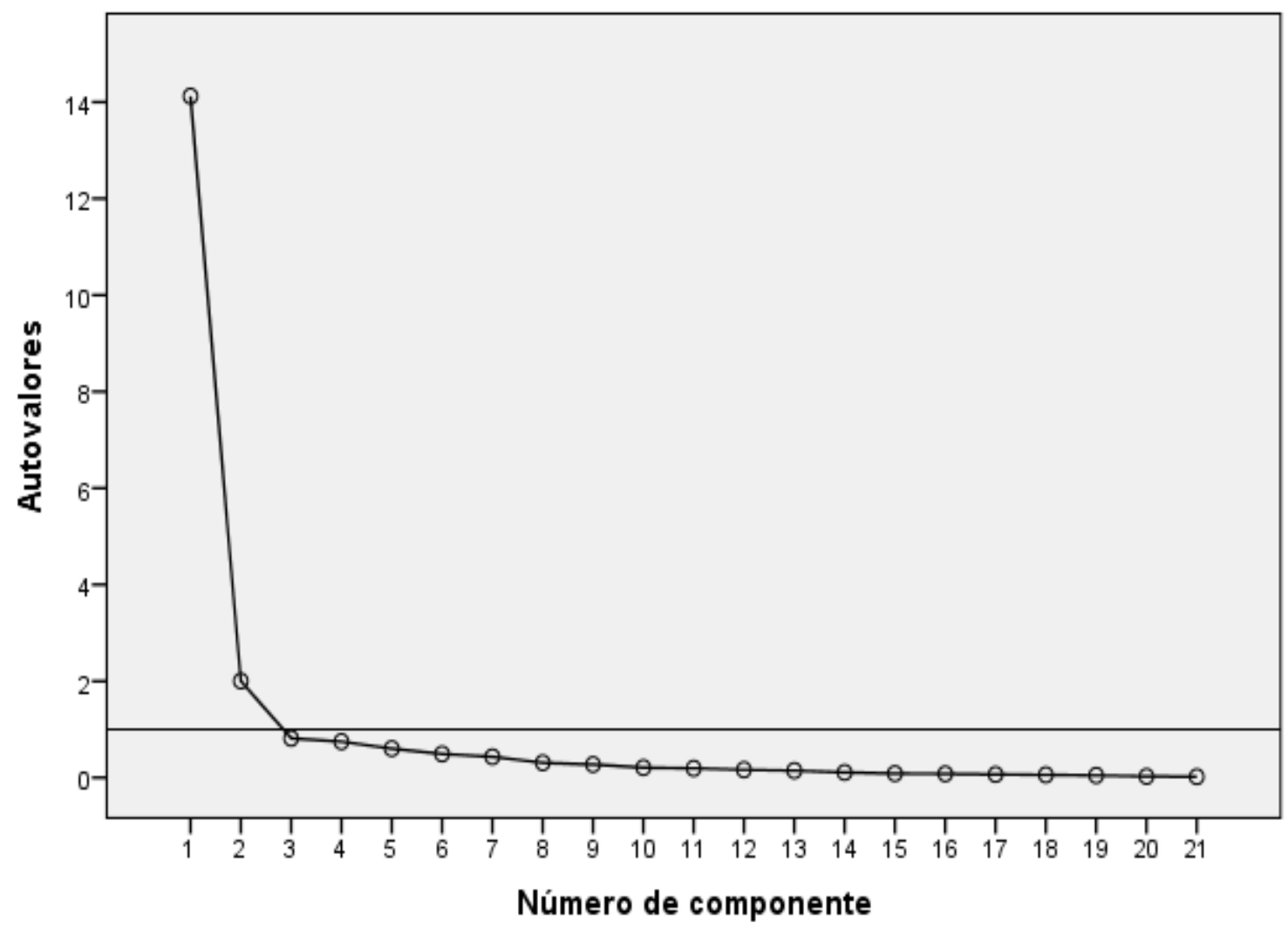

De acuerdo con la regla de Kaiser, en el gráfico de sedimentación, se muestran dos factores que explican la mayor parte de la variabilidad total.

En la varianza total explicada se observa que los primeros dos factores logran explicar $76.78 \%$ de la variabilidad de los datos originales (Tabla 2). 
Construcción y validación de un cuestionario para evaluar la percepción sobre la tutoría metodológica en los cursos de Especialización Médica

Tabla 2. Varianza total explicada.

Table 2. Total variance explained.

\begin{tabular}{|c|c|c|c|c|c|c|c|c|c|}
\hline \multirow{2}{*}{ Componente } & \multicolumn{3}{|c|}{ Autovalores iniciales } & \multicolumn{2}{c|}{$\begin{array}{c}\text { Sumas de las saturaciones al } \\
\text { cuadrado de la extracción }\end{array}$} & \multicolumn{2}{|c|}{$\begin{array}{c}\text { Suma de las saturaciones al cuadrado de } \\
\text { la rotación }\end{array}$} \\
\cline { 2 - 10 } & Total & $\begin{array}{c}\text { \% de la } \\
\text { varianza }\end{array}$ & $\begin{array}{c}\text { \% } \\
\text { acumulado }\end{array}$ & Total & $\begin{array}{c}\text { \% de la } \\
\text { varianza }\end{array}$ & $\begin{array}{c}\text { \% } \\
\text { acumulado }\end{array}$ & Total & $\begin{array}{c}\text { \% de la } \\
\text { varianza }\end{array}$ & \% acumulado \\
\hline 1 & 14.123 & 67.253 & 67.253 & 14.123 & 67.253 & 67.253 & 10.695 & 50.927 & 50.927 \\
2 & 2.002 & 9.533 & 76.786 & 2.002 & 9.533 & 76.786 & 5.430 & 25.859 & 76.786 \\
3 & .815 & 3.883 & 80.669 & & & & & \\
4 & .748 & 3.562 & 84.231 & & & & & \\
5 & .602 & 2.865 & 87.096 & & & & & \\
6 & .491 & 2.340 & 89.436 & & & & & \\
7 & .433 & 2.060 & 91.496 & & & & & \\
8 & .310 & 1.475 & 92.972 & & & & & \\
9 & .272 & 1.295 & 94.267 & & & & & \\
10 & .207 & .987 & 95.254 & & & & & \\
11 & .194 & .926 & 96.180 & & & & & \\
12 & .165 & .787 & 96.966 & & & & & \\
13 & .146 & .697 & 97.663 & & & & & \\
14 & .108 & .515 & 98.179 & & & & & \\
15 & .086 & .408 & 98.587 & & & & & \\
16 & .080 & .383 & 98.971 & & & & & \\
17 & .066 & .314 & 99.284 & & & & & \\
18 & .059 & .283 & 99.567 & & & & & \\
19 & .046 & .220 & 99.788 & & & & & & \\
20 & .026 & .124 & 99.911 & & & & & & \\
21 & .019 & .089 & 100.000 & & & & & & \\
\end{tabular}

Método de extracción: Análisis de Componentes principales.

Extraction method: Principal component analysis.

Con la rotación ortogonal varimax, en la matriz de componentes rotados los ítems se agruparon en dos factores. Además, estos presentan cargas factoriales superiores a 0.55 dentro de cada factor y comunalidades igual o mayores a 0.5 (Tabla 3).

El instrumento final quedo conformado con los 21 ítems propuestos, agrupados en 2 factores o dimensiones. 
Tabla 3. Matriz de componentes rotados.

Table 3. Rotated component matrix.

\begin{tabular}{|c|c|c|c|}
\hline \multirow{2}{*}{ Ítems } & \multicolumn{2}{|c|}{ Componente } & \multirow{2}{*}{ Comunalidades } \\
\hline & 1 & 2 & \\
\hline 12. Ayudó a registrar el protocolo en el CLIES & .883 & & .791 \\
\hline 17. Fomento el debate de ideas con el asesorado & .869 & & .871 \\
\hline 21. Ha resuelto satisfactoriamente cada una de tus dudas & .863 & & .882 \\
\hline 14. Tienen capacidad para orientar en metodología de la investigación & .860 & & .831 \\
\hline 9. Orientó sobre bibliografía a consultar & .836 & & .829 \\
\hline 8. Proporcionó información de buscadores, software, material didáctico & .832 & & .832 \\
\hline 15. Muestra habilidades para el análisis estadístico & .832 & & .751 \\
\hline 18. Propició el análisis critico & .825 & .443 & .877 \\
\hline 7. Facilito la relación con otras personas & .823 & & .774 \\
\hline 21. ¿Estás satisfecho con la asesoría recibida? & .806 & .427 & .831 \\
\hline 11. Proporcionó ayuda inicial para la localización de referencias & .806 & .445 & .848 \\
\hline 10. Capacitó en el uso de software o herramientas que facilitaran la elaboración de la tesis & .775 & .401 & .762 \\
\hline 16. Colaboró en la dirección y redacción del escrito final & .765 & & .651 \\
\hline 20. Domina el tema en el que asesoró & .764 & & .726 \\
\hline 13. Ayudó a realizar la difusión de resultados en foros, congresos y/o publicaciones & .749 & & .612 \\
\hline 2. Creo un clima de confianza & & .889 & .846 \\
\hline 5. Mostró interés en problemas académicos & & .862 & .856 \\
\hline 3. Favoreció una comunicación constante y abierta & & .852 & .857 \\
\hline 1. Empleo frases de motivación & & .802 & .701 \\
\hline 4. Fue respetuoso y atento & & .660 & .500 \\
\hline 6. Permitió que asumieras riesgos y tomaras decisiones en el desarrollo de la tesis & .438 & .556 & .503 \\
\hline
\end{tabular}

Método de extracción: Análisis de componentes principales. Método de rotación: Normalización Varimax con

Kaiser. a. La rotación ha convergido en 3 iteraciones.

Extraction method: Principal component analysis. Rotation method: Varimax with Kaiser normalization. a. The rotation converged in 3 iterations.

Las dos dimensiones del instrumento son:

1) Competencias personales y socioemocionales del tutor, el cual consta de 6 ítems, la puntuación mínima posible es 6 y la máxima 30

2) Competencias para socializar y facilitar proceso investigativo. Consta de 15 ítems, con una puntuación mínima de 15 y máxima de 75.

El Cuestionario para Evaluar la Percepción de la Tutoría Metodológica (CEPTUM) para la elaboración de proyectos de investigación quedo conformado por dos apartados: a) Ficha de identificación del asesorado y del asesor y, b) información sobre el proceso de tutoría metodológica. Esta última conformada por 21 ítems, emplea una escala de Likert, que va desde nunca (1 punto), hasta muy a menudo (5 puntos) de tal forma que la evaluación global de la percepción tiene una puntuación máxima de 105 y mínima de 21. Para la interpretación del instrumento, se consideró que para que la tutoría fuera percibida como aceptable, debe contar con al menos $60 \%$ del total del puntaje total, que corresponderían a 63 puntos, aunque, para conservar la equidistancia entre las distintas categorías se ajustó a al menos $62 \%$, por lo que se propone la 
siguiente escala: Excelente (96 a 105 puntos); muy buena (86 a 95 puntos); buena (76 a 85 puntos); regular (66 a 75 puntos) y mala, menor o igual a 65 puntos.

\section{Discusión}

Se construyó un cuestionario el cual cuenta con dos dimensiones: a) Competencias personales y socioemocionales del tutor y b) Competencias metodológicas para socializar y facilitar proceso investigativo. Tiene como ventaja contar con pocos ítems, lo que facilita su aplicación e interpretación, asimismo, permite tanto a los tutores como a los responsables de los procesos educativos realizar una evaluación continua de este proceso con la finalidad identificar y fortalecer las áreas de oportunidad que se identifiquen. Otra ventaja es que los expertos para la validación, además de tener los conocimientos y experiencia suficiente, también han sido protagonistas de este proceso, tanto en su etapa como residentes, como en la etapa de profesores y de jefes de educación.

Los resultados muestran que el CEPTUM cuenta con adecuadas características psicométricas tanto en su validez de contenido tanto cualitativa (juicio de expertos), como cuantitativa (Índice de Lowshe de 0.99); consistencia interna, mediante el coeficiente de alfa de Cronbach de 0.97 global y en validez de constructo por medio del análisis factorial exploratorio.

Hair (como fue citado por Zamora Muñoz et al., 2010) propone que las variables con comunalidades menores a 0.5 carecen de una explicación suficiente, por ello no deben ser consideradas en la interpretación final del análisis, en nuestro caso, en todos los ítems se supera dicho valor, en consecuencia se consideran representativos de las dimensiones propuestas. Este mismo autor propone ciertas directrices para determinar si una carga factorial es o no significativa, dependiendo del tamaño de la muestra utilizada para el análisis, refiriendo que para un tamaño muestral de 85 se consideran cargas factoriales significativas aquellas iguales o mayores de $0.60 \mathrm{y}$ para uno de 100, la carga significativa debe ser igual o mayor de 0.55. En nuestro caso, donde la muestra fue de 94, los resultados que se obtuvieron cumplen con estos criterios, por lo que se interpreta que existe una correlación significativa entre los factores y las variables que los discriminan.

Otro aspecto a señalar es la presencia de las variables con carga factorial en dos dimensiones, como es el caso de los ítems 10, 11, 18 y 21 de la dimensión Competencias para socializar y facilitar proceso investigativo y del ítem 6 de la dimensión Competencias personales y socioemocionales del tuto, donde estas no son cercanas a cero, sin embargo, en ambos casos al 
ser cargas factoriales menores de 0.55 , no se consideran discriminatorias de ambos componentes, sino del que tiene la carga factorial significativa (Hair citado por Zamora Muñoz et al., 2010).

$\mathrm{Al}$ inicio, en la propuesta del cuestionario se consideraban tres dimensiones para evaluar el proceso de tutoría como lo proponen Haksever y Manisali (2000), al final se observó que los ítems se agrupaban en dos dimensiones, sin que esto afectará la validez teórica del cuestionario.

Además, no hay muchos instrumentos que describan sus procesos de validación y determinación de la consistencia interna que favorezcan una evaluación confiable de él. Si bien el presente cuestionario sólo se enfoca en la tutoría metodológica y no en el proceso global de la tutoría como el instrumento propuesto por De la Cruz y Abreu (2017), consideramos que es un instrumento susceptible de ser usado en los cursos de especialización médica, específicamente en la parte de formación en investigación, la cual representa el área de menos atención, durante el desarrollo de la especialidad médica. Sin embargo, como limitación de este estudio, en un futuro deberá realizarse el análisis factorial confirmatorio, ya que el análisis factorial exploratorio se utiliza para "construir" la teoría y el confirmatorio para "confirmar" la teoría. Este último requerirá una muestra diferente y mayor a 200 participantes (Boomsma y Hoogland citados por BatistaFoguet, et al., 2004).

Como lo refieren Kú y Pool (2018) la evaluación por sí misma no es trascendente, si no va unida de alguna manera al mejoramiento y, para que esto ocurra, es necesario que, sin dejar de ser objetiva, la evaluación incluya un elemento "amigable" de apoyo y consejo. Además de esto, consideramos que, aunque el instrumento es adecuado y confiable, sólo evalúa de manera indirecta las competencias del tutor, ya sea para la tutoría metodológica como para la elaboración de proyectos de investigación, por lo que sugerimos que debe considerarse de alguna manera la evaluación directa de estas competencias que pueden reforzar la validación del instrumento junto con la necesidad del Análisis Factorial Confirmatorio en una muestra mayor.

\section{Agradecimientos}

Agradecemos la participación de cada uno de los voluntarios en este estudio.

\section{Referencias}

Asociación Nacional de Universidades e Instituciones de Educación Superior. (2000). Programas institucionales de tutoría: una propuesta de la ANUIES para su organización y 
Construcción y validación de un cuestionario para evaluar la percepción sobre la tutoría metodológica en los cursos de Especialización Médica

funcionamiento en las instituciones de educación superior. Asociación Nacional de Universidades e Instituciones de Educación Superior.

Academia Mexicana de Cirugía, A. C. (2013). Educación En Salud. (Primera edición). México D.F., México: Alfil S. A. de C. V.

Batista-Foguet, J. M., Coenders, G. \& Jordi Alonso. (2004) Análisis factorial confirmatorio. Su utilidad en la validación de cuestionarios relacionados con la salud. Medicina Clínica (Barcelona), 122(Supp11), 22-27.

Calixto Flores, R. (2016). La tutoría en la formación de maestros. Magister, 28(1), 16-24. DOI: https://doi.org/10.1016/j.magis.2016.07.001

Carretero-Dios, H., \& Perez, C. (2005). Normas para el desarrollo y revisión de estudios instrumentales. International Journal of Clinical and Health Psychology, 5(3), 521-551. http://aepc.es/ijchp/articulos_pdf/ijchp-158.pdf

Castro Rodríguez, Y., Cósar-Quiroz, J., Arredondo-Sierralta, T., \& Sihuay-Torres, K. (2018). Scientific production of theses supported and published by students of Dentistry. Educación Médica, 19, 85-89. DOI: https://doi.org/10.1016/j.edumed.2017.04.002

Castro Rodríguez, Y., Sihuay-Torres, K., \& Perez-Jiménez, V. (2018). Scientific production and perception of research by students of dentistry. Educación Médica, 19(1), 19-22. DOI: https://doi.org/10.1016/j.edumed.2016.11.001

Cruzata-Martínez, A. C.-M., Bellido García, R., Velázquez-Tejeda, M., \& Alhuay-Quispe, J. (2018). La tutoría como estrategia pedagógica para el desarrollo de competencias de investigación en posgrado. Propósitos y Representaciones, 6(2), 09. DOI: https://doi.org/10.20511/pyr2018.v6n2.252

De la Cruz Flores, G., \& Abreu Hernández, L. F. (2017). Evaluación de la tutoría en los estudios d posgrado: construcción y validez de escalas. REDU. Revista de Docencia Universitaria, 15(1), 11. DOI: https://doi.org/10.4995/redu.2017.5682

De La Cruz Flores, G., \& García Campos, T. (2006). Modelo integrador de la tutoría. Revista Mexicana de Investigación Educativa, 11, 1363-1388.

Guerra-Martín, M. D., \& Borrallo-Riego, Á. (2018). Tutoring and academic performance from the perspective of Health Sciences students and teachers. A systematic review. Educación Médica, 19(5), 301-308. DOI: https://doi.org/10.1016/j.edumed.2017.03.019 
Haksever, A. M., \& Manisali, E. (2000). Assessing supervision requirements of PhD students: The case of construction management and engineering in the UK. European Journal of Engineering Education, 25(1), 19-32. DOI: https://doi.org/10.1080/030437900308616

Hamui-Sutton, A., Enríquez-López, P., Hernández-Becerril, C., Lavalle-Montalvo, C., \& VilarPuig, P. (2018). What do residents think about their tutors? A qualitative approach. Educación Médica, 19(1), 9-18. DOI: https://doi.org/10.1016/j.edumed.2016.11.003

Herrera-Márquez, J. R., \& González-Cabello, H. J. (2015). Investigación en pediatría. Revista Médica Del Instituto Mexicano Del Seguro Social, 53(3), S228-S229. http://www.redalyc.org/articulo.oa?id=457744943001

IMSS. (2012). La educación en salud en el IMSS (C. Q. S. A. V. Á. Echevarría Zuno Santiago, Lifshitz Alberto (ed.); Primera). Instituto Mexicano del Seguro Social.

Kú Hernández, O. E., \& Pool Cibrián, W. J. (2018). Construcción y Validación de Instrumentos para la Evaluación de la Práctica Pedagógica en Educación Básica del Estado de Yucatán. Revista Iberoamericana de Evaluación Educativa, 11(1), 23-41. DOI: https://doi.org/10.15366/riee2018.11.1.002

López Santiago, A., Baeza López, J. M., \& Lebrato García, R. M. (2000). Qué esperan de sus tutores los residentes de medicina de familia? Una aproximación cualitativa. Atención Primaria / Sociedad Española de Medicina de Familia y Comunitaria, 26(6), 362-367. DOI: https://doi.org/10.1016/S0212-6567(00)78684-7

Rosas, A. K., Flores, D., \& Valarino, E. (2006). Rol del tutor de tesis: competencias, condiciones personales y funciones. Investigación y Postgrado. 21(1), 153-186. https://dialnet.unirioja.es/servlet/articulo?codigo=2309849

Ruiz, C. (2013). Instrumentos y Técnicas de Investigación Educativa-Carlos Ruiz-Bolívar (3a Ed.) https://www.academia.edu/37886948/Instrumentos_y_Tecnicas_de_Investigación_Educat $\underline{\text { iva_-_Carlos_Ruiz-Bolivar.pdf }}$

Zamora Muñoz, S., Monroy Cazorla, L., \& Chávez Álvarez, C. (2010). Análisis factorial: una técnica para evaluar la dimensionalidad de las pruebas. In Centro Nacional de Evaluación para la Educación Superior, A.C. (Ceneval). (Primera). CENEVAL. https://www.ceneval.edu.mx/documents/20182/0/CuadernoTecnico061aed.pdf/a922a29593f3-4bd0-9c8b-28a52c499cf5 